\title{
Azide-based Cross-Linking of Polymers of Intrinsic Microporosity (PIMs) for Condensable Gas Separation ${ }^{a, b}$
}

\author{
Naiying Du, Mauro M. Dal- Cin, Ingo Pinnau, Andrzej Nicalek, \\ Gilles P. Robertson, Michael D. Guiver*
}

Cross-linked polymers of intrinsic microporosity (PIM)s for gas separation membranes, were prepared by a nitrene reaction from a representative PIM in the presence of two different diazide cross-linkers. The reaction temperature was optimized using TGA. The homogenous membranes were cast from THF solutions of different ratios of PIM to azides. The resulting cross-linked structures of the PIMs membranes were formed at $175^{\circ} \mathrm{C}$ after $7.5 \mathrm{~h}$ and confirmed by TGA, XPS, FT-IR spectroscopy and gel content analysis. These resulting cross-linked polymeric membranes showed excellent gas separation performance and can be used for $\mathrm{O}_{2} / \mathrm{N}_{2}$ and $\mathrm{CO}_{2} / \mathrm{N}_{2}$ gas pairs, as well as for condensable gases, such as $\mathrm{CO}_{2} / \mathrm{CH}_{4}$, propylene/propane separation. Most importantly, and differently from typical gas separation membranes derived from glassy polymers, the crosslinked PIMs showed no obvious $\mathrm{CO}_{2}$ plasticization up to $20 \mathrm{~atm}$ pressure of pure $\mathrm{CO}_{2}$ and $\mathrm{CO}_{2} / \mathrm{CH}_{4}$ mixtures.

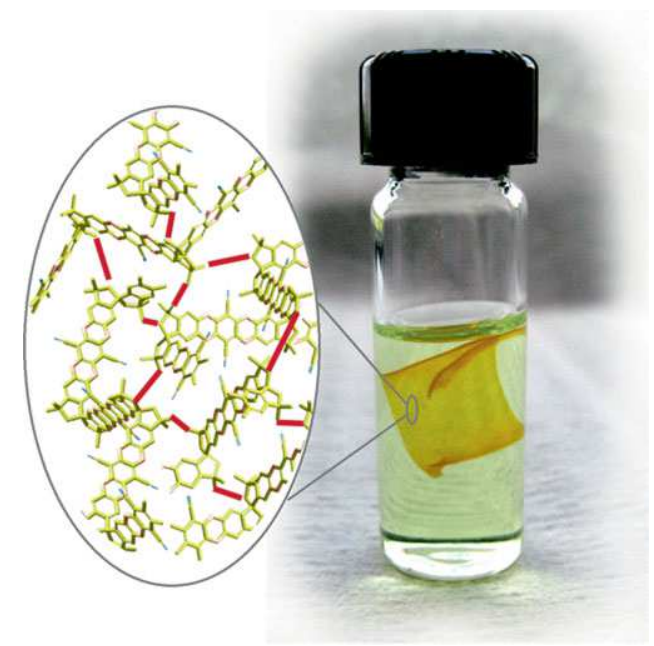

N. Du, M. M. D. Cin, A. Nicalek, G. P. Robertson, M. D. Guiver Institute for Chemical Process and Environmental Technology, National Research Council of Canada, Ottawa, Ontario, K1A oR6, Canada

E-mail: Michael.Guiver@nrc-cnrc.gc.ca

I. Pinnau

Advanced Membranes and Porous Materials Center, King

Abdullah University of Science and Technology, Thuwal 239556900 , Kingdom of Saudi Arabia

M. D. Guiver

WCU Department of Energy Engineering, Hanyang University, 17 Haengdang-dong, Seongdong-gu, Seoul 133-791, South Korea

\footnotetext{
$\mathrm{a}$ 을 Supporting Information is available at Wiley Online Library or from the author.

${ }^{b}$ NRCC No. 52826
}

\section{Introduction}

Membrane technology has become increasingly attractive for gas separation due to its economical and environmental advantages, such as lower cost, reduced energy consumption, portability and modular nature, making it valuable for offshore and remote-location gas processing. ${ }^{[1]}$ During the last decade in the industrial gas separations sector, there has been a continued interest in further improving technology for the separation or purification of mixtures containing condensable gases. ${ }^{[2,3]}$ However, a major technological hurdle to industrial membrane separations involving condensable gases at high pressures is plasticization. Plasticization leads to membrane swelling, resulting in a significant increase in the permeabilities of all 
components in the feed and a decrease in the gas selectivities. Although numerous research articles and patents describe new high-performance polymers (e.g., polyimides, polysulfones, polycarbonates, polyethers, polyamides, polyarylates, polypyrrolones, etc.) with desirable gas separation properties, ${ }^{[4]}$ the selectivities of mixed gases, which contain at least one condensable gas component under high pressure conditions are generally much lower than expected, due to plasticization. Since 2004, a novel class of polymers for gas separation, termed 'polymers of intrinsic microporosity' (PIMs), which reveal unusually high free volume and high surface areas, have been reported by Budd and McKeown. ${ }^{[5,6]}$ Different from other polymeric microporous materials or zeolites and activated carbons, which are widely used industrially, PIMs have special ladder-type main chain structures with contorted or kinked centers which prevent efficient space packing, resulting in an intrinsically microporous structure. In addition, PIMs have another attractive advantage of good processability: high molecular weight PIMs are soluble in numerous solvents and can be readily cast into membranes or films. A number of published examples of PIMs have shown single gas permeability data that are located around the Robeson upper bound for the separation of important gas pairs $\left(\mathrm{O}_{2} / \mathrm{N}_{2}, \mathrm{CO}_{2} / \mathrm{N}_{2}\right.$, and $\left.\mathrm{CO}_{2} / \mathrm{CH}_{4}\right)$. PIMs characteristically have moderate to good selectivity and very high permeability. ${ }^{[7-9]}$ However, PIMs cannot maintain good performance at high pressures in the presence of condensable gases mixtures, such as propylene/propane and $\mathrm{CO}_{2} / \mathrm{CH}_{4}$. This behaviour is similar to typical glassy polymers used for gas separation processes operating by the solution-diffusion mechanism, which usually suffer from plasticization of the stiff polymer matrix by the sorbed penetrant molecules. ${ }^{[10]}$ In view of the outstanding properties of PIMs, and the potential for their use in industrial gas separations, there is a strong need to develop new plasticization-resistant PIM membrane materials. Plasticization is generally defined as an increase in the segmental motion of polymer chains, due to the presence of one or more sorbents. Consequently, the permeability of both components increases and the selectivity decreases. ${ }^{[11]}$ The loss in selectivity for condensable gases is mainly caused by a reduction in the diffusivity selectivity due to excessive segmental motion ${ }^{[12]}$ Conventional methods for stabilizing polymeric membranes are either annealing or crosslinking. Cross-linking is a useful method to increase membrane stability in the presence of aggressive feed gases and to simultaneously reduce plasticization of the membrane. Cross-linking methods for polymer membranes include thermal treatment, radiation, chemical crosslinking, UV-photochemical, etc. Several investigations have shown that cross-linking of polymeric gas separation membranes could improve separation characteristics by decreasing plasticization. ${ }^{[13-17]}$ However, most of the preceding cross-linking methods involve procedures that would be difficult to apply in commercial membrane manufacturing processes. For example, excessive heat treatment significantly reduces the membrane $\mathrm{CO}_{2}$ permeability. Furthermore, the high temperatures that are sometimes required $\left(>350^{\circ} \mathrm{C}\right)$ make this approach less appealing for large-scale membrane production.

Previously reported work on network-PIMs with interesting metal-containing phthalocyanine or porphorinelike structures has shown that they have potential as adsorbents and catalyst supports. ${ }^{[7]}$ The present work pertains to plasticization-resistant chemically cross-linked PIMs via a nitrene reaction. A representative polymer, PIM1 , was selected, since it is the most widely reported and characterized ladder-type PIM. However this method could be equally applicable to many other PIM structures. The structure of PIM-1 is shown in Scheme 1, and is derived from the step-growth polycondensation of $5,5^{\prime}, 6,6^{\prime}$-tetrahydroxy-3,3,3',3'-tetramethylspirobisindane and 2,3,5,6tetrafluoroterephthalonitrile.

After film formation from PIM-1 / azide precursors, crosslinked PIMs membranes with excellent gas separation performance were prepared by heating under vacuum at $175^{\circ} \mathrm{C}$ for $7.5 \mathrm{~h}$. To maintain the film morphology and minimize production costs, mild processing temperatures are required ( $<180{ }^{\circ} \mathrm{C}$ preferably). Unlike many previously reported chemical cross-linking methods based on crosslinking reagents, non-solvent treatment and heat treatment, the cross-linking reaction temperatures tend to be<smiles>CC1(C)Cc2cc3c4cc2Oc2cc5c(cc2C(C)(C)C(C)(C)CC4(CC3(C)C)C(C)(C)O1)Oc1c(C#N)c2c(c(C#N)c1C5(C)C)C(C)(C)C(C)(C)O2</smiles>

PIM-1<smiles>Nc1ccc(S(=O)(=O)c2ccc(N)cc2)cc1</smiles>

azide1

4-azido phenyl sulfone<smiles>CC1C/C(=C\c2ccc(N=[W])cc2)C(=O)/C(=C/c2ccc([N+]#N)cc2)C1</smiles>

\section{2,6-bis(4-azidobenzylidene)-4-methylcyclohexanone}

Scheme 1. Structures of PIM-1, azide1 and azide2. 
lower with diazides. ${ }^{[18-20]}$ While diazides have also been previously utilized to cross-link polymers for gas separation membranes, ${ }^{[21]}$ the present PIM-1 / azide systems form unique polymer networks having considerable free volume and have excellent gas permeability / selectivity properties, particularly for gas pairs containing condensable gases. Azides polymerize in situ within and throughout the PIM-1 phase and thus stabilize the multiphase system. Consequently, phase separation that may arise from the mutual incompatibility inherent in multi-polymer systems at the macroscale level can be avoided during the network formation. ${ }^{[22]}$ The gas separation properties of the resulting robust plasticization-resistant PIM membrane materials were investigated.

\section{Results and Discussion}

The use of appropriate polymeric precursors is the first step for preparing cross-linked membranes. Although pretreatment and cross-linking conditions are also crucial issues in determining the membrane performance, the chemical structure and physical properties of polymer precursors should be considered foremost. Based on the variety of high molecular weight PIMs reported in the literature, ${ }^{[6,23-26]}$ PIM-1 (Scheme 1) was selected as the representative polymer in this class of materials, since it is the most widely reported and characterized, due to its simple preparation from commercial monomers, high fractional free volume (FFV), high thermal stability, good processability and mechanical properties. Two diazides (Scheme 1) that are capable of thermal curing were investigated as cross-linking reagents to form the crosslinked PIM. These two diazides are negative-type thermally activated or photo-sensitive compounds. The thermal decomposition of the diazides proceeds with an initial degradation of the $-\mathrm{N}-\mathrm{N} \equiv \mathrm{N}$ 'single' bond, releasing nitrogen gas $\left(\mathrm{N}_{2}\right)$ and leaving behind highly reactive divalent nitrene $-\ddot{\mathbf{N}}$ : groups. The nitrene group can initiate addition reactions with the alkyl $\mathrm{C}-\mathrm{H}$ to form a covalent network. Besides creating chemical linkages between nitrene groups and alkyl $\mathrm{C}-\mathrm{H}$ on PIM-1 polymer chains (such as $>\mathrm{CH}_{2}$ and $-\mathrm{CH}_{3}$ ), one of the diazides 2,6-bis(4azidobenzylidene)-4-methyl-cyclohexanone (azide2 in Scheme 1) is also capable of reacting with itself to form a network through the nitrene and $\mathrm{C}=\mathrm{C}$ bond. Hence, under a variety of different reaction conditions, such as reaction time, temperature and reagent ratios, PIM-1 / azide membranes are capable of forming cross-linked or polymer network structures.

The reaction temperature was investigated and optimized using thermogravimetric analysis (TGA). The TGA curves of PIM-1 / azide1 and PIM-1 / azide2 (mol\%= 80:20) from 100 to $600{ }^{\circ} \mathrm{C}$ are quite similar. At the first decom- position stage between $\approx 140{ }^{\circ} \mathrm{C}$ and $200^{\circ} \mathrm{C}$, approximately $2.5-3.0$ wt.-\% loss was observed for the mixtures, which corresponds to almost complete decomposition compared with the calculated response for decomposition of $-\mathrm{N}_{3}$ groups in PIM-1 / azide $(\mathrm{mol} \%=80: 20)$. A relatively gradual degradation rate with increasing temperature was maintained during the whole process. The lower the ratio of the diazide: PIM in the mixtures, the smaller the weight loss that was observed. The cross-linked PIM-1 / azides were further characterized by TGA and DSC, and the results compared to the polymeric nitrile precursor - PIM-1. As with PIM-1, none of the cross-linked polymer films had a discernable $T_{\mathrm{g}}$ in the measured range up to $350^{\circ} \mathrm{C}$, likely due to the absence of rotational freedom of linkages in the main chain. The resulting cross-linked matrix had a lower thermal stability than the pure PIM-1, which begins to decompose at around $430^{\circ} \mathrm{C}$. As a result, for the cross-linked PIM-1 / azide precursors, the initial weight loss occurring before $430^{\circ} \mathrm{C}$ is mainly attributable to the decomposition of $-\mathrm{N}-\mathrm{C}$, which resulted from the nitrene reaction. The weight loss occurring after $430^{\circ} \mathrm{C}$ is caused mainly by the decomposition of the polymer backbone of PIM-1. Details are included in the Supporting Information, Fig. 1. From the TGA data, one can conclude that the pure PIM-1 polymer is a suitable precursor for making cross-linked membranes utilizing the nitrene reaction.

The cross-linked structures were characterized by FT-IR spectroscopy and XPS. The membranes cast from solutions of PIM-1 with azide1 and 2 (PIM-1 / azides, mol\%=80:20) show the characteristic absorption band of azides at $2100 \mathrm{~cm}^{-1}$, as shown in the Supporting Information, Fig. 2. After a 7.5-hour cross-linking reaction at $175^{\circ} \mathrm{C}$, the relative intensity of the azide absorption bands decreased obviously compared with other bands. Other characteristic absorption bands observed in PIM-1 / azide2 in the range of $1650-1690 \mathrm{~cm}^{-1}$, corresponding to $\mathrm{C}=\mathrm{C}$ stretching vibrations, almost disappeared after a 7.5 -h reaction, indicating that most of the $\mathrm{C}=\mathrm{C}$ groups of azide 2 reacted.

XPS offers another useful spectroscopic method to provide evidence of cross-linking. High-resolution spectra (Supporting Information Fig. 3) of the nitrogen 1s binding energies (BE) of diazides revealed two distinct peaks, one centered at $401 \mathrm{eV}$ and another centered at $404 \mathrm{eV}$, with a 2:1 ratio of the peak areas. ${ }^{[27]}$ The less intense, higher energy, $404 \mathrm{eV}$ peak is unusual for covalently-bonded nitrogen, and we assign this peak to the electron-deficient nitrogen in the azide group. Reaction of the diazide with PIM-1 via the nitrene reaction led to a considerable reduction in intensity of the $404 \mathrm{eV}$ peak. The nitrogen $1 \mathrm{~s}$ signal at $401 \mathrm{eV}$ broadened, indicating the presence of chemically distinct nitrogen atoms. Except for these two small peaks, a large peak at $399 \mathrm{eV}$ is attributed to $-\mathrm{C} \equiv \mathrm{N}$ groups present on the PIM-1 chain, indicating the reaction primarily did not occur through the nitrile group. Assuming

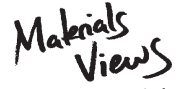

www.MaterialsViews.com
Macromol. Rapid Commun. 2011, 32, 631-636 (c) 2011 WILEY-VCH Verlag GmbH \& Co. KGaA, Weinheim 
that azide disappearance is correlated to product formation, these results provide additional evidence that cross-linking occurred.

The cross-linked structure was also analyzed for gel content. PIM-1 can be readily dissolved in chloroform, THF, dichloromethane etc. Different from PIM-1, the resulting cross-linked PIM-1 / azides films were almost completely insoluble in chloroform (see Supporting Information Fig. 4). It was found that azide2 exhibited better cross-linking performance than azide1, presumably because it can crosslink with itself through the double bonds via nitrene, whereas azide1 has no double bonds that allow this to occur. Cross-linked PIM-1 / azide1 (PIM-1 / azide1, mol\% = 80: 20 ) has $\approx 80 \%$ gel content remaining and cross-linked PIM-1 / azides2 (mol\%=80 : 20) has $>95 \%$ gel content remaining. The results indicate that the nitrene reaction generated by diazides in the presence of PIM-1 led to an insoluble cross-linking network.

Free-standing flexible films of the cross-linked PIM-1 / azides with different ratios of PIM-1 and azides were prepared for gas separation testing. Single-gas permeability coefficients $(P)$ for $\mathrm{O}_{2}, \mathrm{~N}_{2}, \mathrm{CH}_{4}, \mathrm{CO}_{2}$, propane, propylene and $\mathrm{CO}_{2} / \mathrm{CH}_{4}$ mixtures were determined at $25^{\circ} \mathrm{C}$ for dense, isotropic films and a summary of these $P$ values and selectivities for selected gas pairs are shown in Figure 1 to 3 .

Based on a previous aging study we conducted, PIM-1 membranes undergo a significant decline in permeability when exposed to condensable gases during the first $100 \mathrm{~d}$ of testing; for example, the permeability declined to about $60 \%$ for $\mathrm{CO}_{2}$ and $40 \%$ for $\mathrm{CH}_{4}$, respectively. Thereafter, the aging rate slowed down and the permeabilities only varied $5-10 \%$ in the following $350 \mathrm{~d}$. Annealing is another approach for stabilizing polymeric membranes. Hence, preaged PIM-1 (PIM-1*) and annealed PIM-1 (PIM-1 $\left.{ }^{\mathrm{A}}\right)$ were

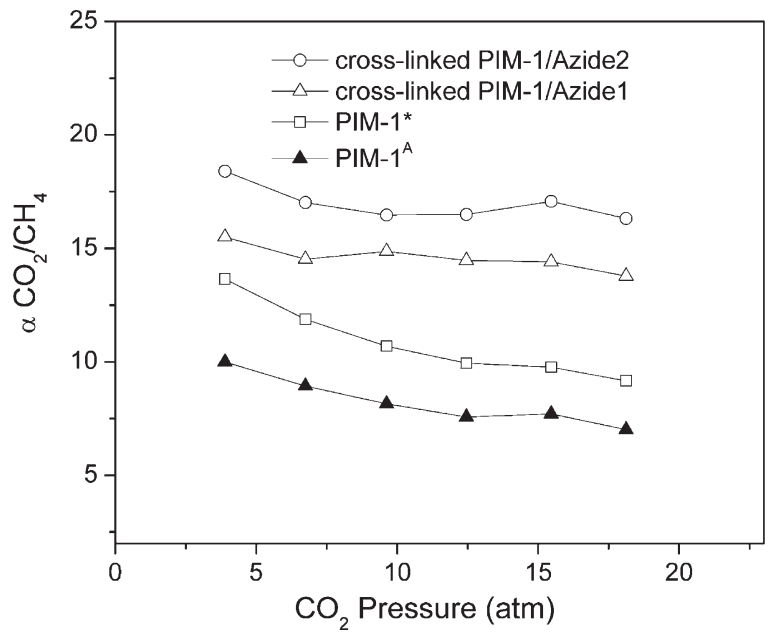

Figure 1. Relationship between pressure and pure gas $\mathrm{CO}_{2} / \mathrm{CH}_{4}$ selectivity for cross-linked PIM-1 / azides $(\mathrm{mol} \%=80: 20)$, $\mathrm{PIM}-1^{*}$ and $\mathrm{PIM}-1^{\mathrm{A}}$.

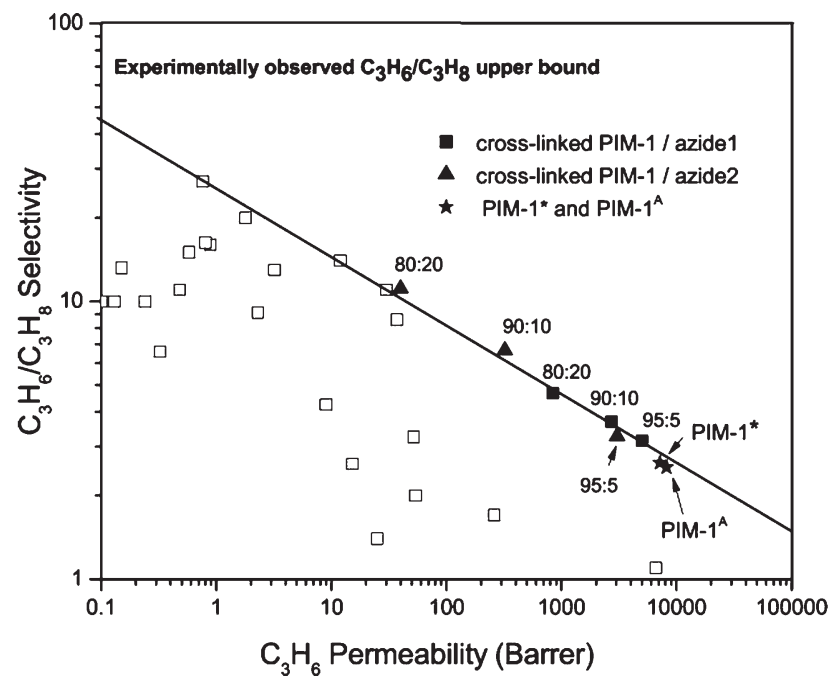

Figure 2. Propylene permeability and pure gas propylene/propane selectivity for various compositions of cross-linked PIM-1 / azide membranes in comparison with reported data.

selected as reference membranes for the cross-linked membranes, rather than the newly-fabricated unannealed PIM-1 membrane. In comparison with PIM-1 ${ }^{\mathrm{A}}$, which was annealed under the same conditions as those that were made by the nitrene reaction, the cross-linked PIM-1 / azides exhibited higher selectivities, coupled with expected reductions in gas permeabilities. Figure 1 shows that

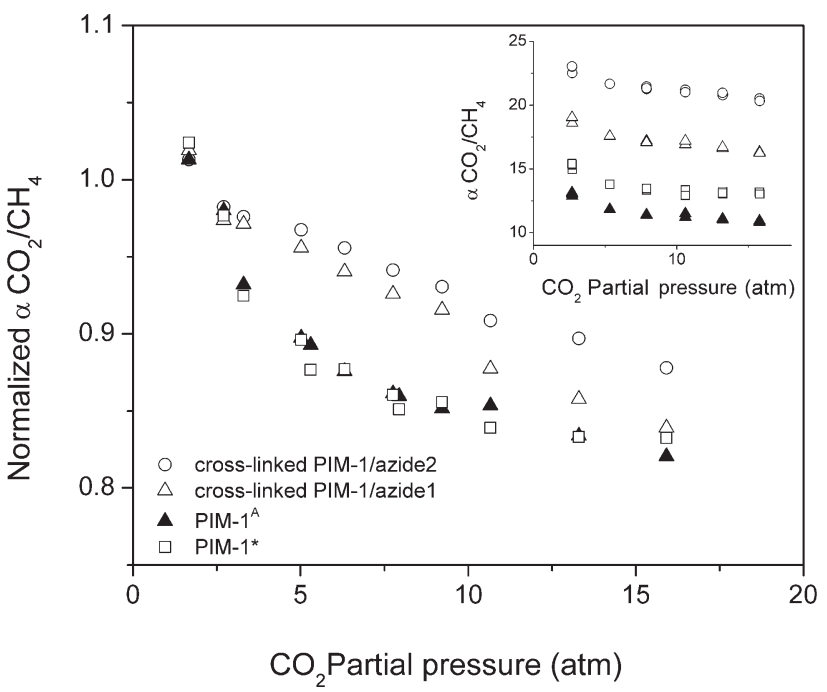

Figure 3. Effect of $\mathrm{CO}_{2}$ partial pressure on mixed gas $\mathrm{CO}_{2} / \mathrm{CH}_{4}$ selectivity in cross-linked PIM-1 / azide1, cross-linked PIM-1 / azide2 (mol\% $=80: 20$ ), $\mathrm{PIM}-1^{*}$ and $\mathrm{PIM}-1^{\mathrm{A}}$ at $25^{\circ} \mathrm{C}$, Normalized mixed gas selectivity vs. pressure was derived from mixed gas selectivity vs. pressure (inset). The mixed gas $\mathrm{CO}_{2} / \mathrm{CH}_{4}$ feed compositions (in mol\% $\mathrm{CO}_{2}$ : $\mathrm{mol} \% \mathrm{CH}_{4}$ ) were 50:50 and 80:20. $\mathrm{CO}_{2} / \mathrm{CH}_{4}$ mixed gases were tested at 3.4, 6.8, 10.2, 13.6 and $17 \mathrm{~atm}$., corresponding to $1.7,3.4,5.1,6.8$ and $8.5 \mathrm{CO}_{2}$ partial pressures for the 50:50 composition and 2.72, 5.44, 8.16, 10.88 and $13.6 \mathrm{~atm} . \mathrm{CO}_{2}$ partial pressures for the 80:20 composition. 
compared to PIM-1, the azide-based cross-linked PIMs have much higher selectivity for pure $\mathrm{CO}_{2} / \mathrm{CH}_{4}$ gas pair separation under the different pressures. The selectivities for pure $\mathrm{O}_{2} / \mathrm{N}_{2}$ and $\mathrm{CO}_{2} / \mathrm{N}_{2}$ were in the range of 3.3-4.8 and 16-27, respectively (see Supporting Information Table 1). These results agree with the general trade-off tendency for selectivity - gas permeability for polymer membranes, i.e. higher $\mathrm{O}_{2}$ and $\mathrm{CO}_{2}$ permeability is gained at the cost of lower selectivity and vice versa. In addition, the value of the annealed PIM-1 ${ }^{\mathrm{A}}$ was almost the same as the PIM- $1^{*}$ and half the values of PIM-1 typically reported before, ${ }^{[8]}$ but coupled with a corresponding increase in selectivity (Figure 1).

An experimentally observed upper bound plot for $\mathrm{C}_{3} \mathrm{H}_{6} /$ $\mathrm{C}_{3} \mathrm{H}_{8}$ separation, constructed using available literature data, was reported previously. ${ }^{[28]}$ Compared to the majority of previously reported polymers, cross-linked PIM-1 / azides exhibit high permeabilities coupled with moderate selectivities that combine to give values that are close to, or even exceed, the experimentally observed upper bound for the $\mathrm{C}_{3} \mathrm{H}_{6} / \mathrm{C}_{3} \mathrm{H}_{8}$ gas pair (Figure 2). These results suggest cross-linked PIM-1 / azide membranes have potential for the industrially important separation of $\mathrm{C}_{3} \mathrm{H}_{6} / \mathrm{C}_{3} \mathrm{H}_{8}$, and that the gas transport properties can be tuned by controlling the cross-linking parameters.

Glassy polymers, such as polyimides, typically exhibit substantial decreases in $\mathrm{CO}_{2} / \mathrm{CH}_{4}$ selectivity under mixedgas conditions, due to $\mathrm{CO}_{2}$ swelling. $\mathrm{CO}_{2}$ acts as a plasticizer causing the permeability of $\mathrm{CH}_{4}$ to increase more relative to $\mathrm{CO}_{2}$, resulting in a decrease in selectivity. ${ }^{[29]}$ The $\mathrm{CO}_{2} / \mathrm{CH}_{4}$ selectivity for the cross-linked PIM-1 / azide polymer membranes decreased to a lesser degree than PIM-1 ${ }^{\mathrm{A}}$ and PIM-1* for mixed gases at high $\mathrm{CO}_{2}$ partial pressure (up to $\sim 20 \mathrm{~atm}$ ) (Figure 3), which indicates that plasticization was suppressed. The individual contributions of pressure dependant pure gas permeabilities of $\mathrm{CO}_{2}$ and $\mathrm{CH}_{4}$ for Figure 1 are shown in the Supporting Information Fig. 5. In summary, cross-linked PIM-1 / azide polymer membranes show resistance to plasticization at high $\mathrm{CO}_{2}$ partial pressures.

\section{Conclusion}

Membranes with cross-linked structures derived from a combination of PIM-1 and diazide precursors were successfully prepared via a low-temperature nitrene reaction. This method is based on highly reactive nitrene groups generated in situ that react with the alkyl $>\mathrm{CH}_{2}$ in the PIM-1 spirobisindane segment. In the case of one of the diazides, the nitrene may also react with the $-\mathrm{C}=\mathrm{C}-$ bond of the cross-linking agent. The azide-based nitrene reaction with PIM-1 is distinct from the [2+3] cycloaddition reaction of azide with the nitrile group of PIM-1 leading to a tetrazole functionality, which we recently reported for $\mathrm{CO}_{2}$ separation applications. ${ }^{[30]}$ The gas separation properties of the cross-linked polymers can be tuned by the selection and concentration of diazide and the degree of cross-linking. In comparison with PIM-1 ${ }^{\mathrm{A}}$ and PIM-1* ${ }^{*}$, crosslinked PIM-1 / azides, prepared under these reaction conditions, exhibited higher $\mathrm{O}_{2} / \mathrm{N}_{2}, \mathrm{CO}_{2} / \mathrm{CH}_{4}$ and $\mathrm{CO}_{2} / \mathrm{N}_{2}$ selectivities, coupled with expected reductions in gas permeabilities. In addition, cross-linked PIM-1 / azides exhibit high permeability coupled with moderate selectivity with values close to, or exceeding the experimentally observed $\mathrm{C}_{3} \mathrm{H}_{6} / \mathrm{C}_{3} \mathrm{H}_{8}$ upper bound. The results of the mixed gas $\mathrm{CO}_{2} / \mathrm{CH}_{4}$ experiments indicate that cross-linked PIMs exhibit a reduction of plasticization in the presence of condensable gases.

Acknowledgements: The authors are grateful to David Kingston of the National Research Council for the XPS measurements. MDG acknowledges partial support from the WCU (World Class university) program through the National Research Foundation of Korea funded by the Ministry of Education, Science and Technology (No. R31-2008-000-10092-0).

Received: December 8, 2010; Revised: February 3, 2011; Published online: March 11, 2011; DOI: 10.1002/marc.201000775

Keywords: azide; crosslinked polymer; gas permeability; gas selectivity, microporous polymer; PIM

[1] S. I. Semenova, J. Membr. Sci. 2004, 231, 189.

[2] K. Okamoto, K. Noborio, J. Hao, K. Tanaka, H. Kita, J. Membr. Sci. 1997, 134, 171.

[3] R. A. Meyers, Handbook of petroleum refining processes, New York McGraw-Hill, 1986, p. 10.01.

[4] W. J. Koros, G. K. Fleming, J. Membr. Sci. 1993, 83, 1.

[5] P. M. Budd, B. S. Ghanem, S. Makhseed, N. B. McKeown, K. J. Msayib, C. E. Tattershall, Chem. Commun. 2004, 230.

[6] P. M. Budd, E. S. Elabas, B. S. Ghanem, S. Makhseed, N. B. McKeown, K. J. Msayib, C. E. Tattershall, D. Wong, Adv. Mater. 2004, 16, 456.

[7] [7a] N. B. McKeown, P. M. Budd, K. J. Msayib, B. S. Ghanem, H. J. Kingston, C. E. Tattershall, S. Makhseed, K. J. Reynolds, D. Fritsch, Chem. Eur. J. 2005, 11, 2610; [7b] P. M. Budd, B. Ghanem, K. Msayib, N. B. McKeown, C. Tattershall, J. Mater. Chem., 2003, 13, 2721.

[8] N. Du, G. P. Robertson, J. Song, I. Pinnau, S. Thomas, M. D. Guiver, Macromolecules, 2008, 41, 9656.

[9] S. Thomas, I. Pinnau, N. Du, M. D. Guiver, J. Membr. Sci. 2009, $333,125$.

[10] WO 2010008660A1 (2010), UOP LLC, C. Liu, S. T. Wilson, D. A. Lesch, High Plasticization-Resistant Cross-Linked Polymeric Membranes for Separations.

[11] W. J. Koros, M. W. Hellums, Encyclopedia of Polymer Science and Engineering; John Wiley and Sons, New York 1989, Suppl. Vol., p 724.

[12] C. Staudt-Bickel, W. J. Koros, J. Membr. Sci. 2000, 170, 205.

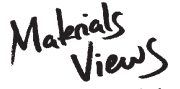

www.MaterialsViews.com 
[13] A. Bos, I. G. M. Punt, M. Wessling, H. Strathmann, Sep. Purif. Technol. 1998, 14, 27.

[14] M. E. Rezac, E. T. Sorensen, H. W. Beckman, J. Membr. Sci. 1997, 136, 249.

[15] M. E. Rezac, B. Schoberl, J. Membr. Sci. 1999, 156, 211.

[16] J. D. Wind, C. Staudt-Bickel, D. R. Paul, W. J. Koros, Macromolecules 2003, 36, 1882.

[17] N. P. Patel, A. C. Miller, R. J. Spontak, Adv. Func. Mater. 2004, 14, 699.

[18] P. S. Tin, T. S. Chung, Macromol Rapid Commun. 2004, 25, 1247.

[19] P. S. Tin, T. S. Chung, S. Kawi, M. D. Guiver, Microporous Mesoporous Mater. 2004, 73, 151.

[20] Y. Xiao, T. S. Chung, H. M. Guan, M. D. Guiver, J. Membr. Sci. 2007, 302, 254.

[21] [21a] S. D. Kelman, B. W. Rowe, C. W. Bielawski, S. J. Pas, A. J. Hill, D. R. Paul, B. D. Freeman, J. Membr. Sci. 2008, 320, 123; [21b] L. Shao, J. Samseth, M. Hägg, J. Membr. Sci. 2009, 326,
285; [21c] M. L. Chng, Y. Xiao, T.-S. Chung, M. Toriida, S. Tamai, Carbon, 2009, 47, 1857.

[22] L. H. Sperling, V. Mishra, Polym. Adv. Tech. 1996, 7, 197.

[23] N. Du, G. P. Robertson, I. Pinnau, M. D. Guiver, Macromolecules. 2009, 42, 6023.

[24] N. Du, G. P. Robertson, I. Pinnau, M. D. Guiver, Macromolecules 2010, 43, 8580

[25] N. Du, G. P. Robertson, I. Pinnau, S. Thomas, M. D. Guiver, Macromol. Rapid Commun. 2009, 30, 584.

[26] N. Du, G. P. Robertson, J. Song, I. Pinnau, M. D. Guiver, Macromolecules. 2009, 42, 6038.

[27] E. W. Wollman, D. Kang, C. D. Frisbie, I. M. Lorkovic, M. S. Wrighton, J. Am. Chem. Soc. 1994, 116, 4395.

[28] R. L. Burns, W. J. Koros, J. Membr. Sci. 2003, 211, 299.

[29] A. Bos, I. G. M. Pünt, M. Wessling, H. Strathmann, J. Membr. Sci. 1999, 155, 67.

[30] N. Du, H. B. Park, G. P. Robertson, M. M. Dal-Cin, T. Visser, L. Scoles, M. D. Guiver, Nature Mater. DOI: 10.1038/nmat2989. 